\title{
A Case Report on Atypical Presentation of Cerebral Venous Sinus Thrombosis, a Young Adult with Recurrent Fall: A Clinical Quandary
}

\author{
Aishwarya Nandy ${ }^{1}$, Sayani Banerjee ${ }^{2}$, Rajesh Gupta ${ }^{3}$ \\ ${ }^{1}$ Department of Emergency Medicine, Ramkrishna Care Hospital, Raipur, India \\ ${ }^{2}$ Department of Emergency Medicine, Calcutta Medical Research Institute, Kolkata, India \\ ${ }^{3}$ Department of General Medicine, Ramkrishna Care Hospital, Raipur, India \\ Email: aishwarya_nandy@yahoo.com,sayani.dr@gmail.com
}

How to cite this paper: Nandy, A., Banerjee, S. and Gupta, R. (2021) A Case Report on Atypical Presentation of Cerebral Venous Sinus Thrombosis, a Young Adult with Recurrent Fall: A Clinical Quandary. Open Journal of Emergency Medicine, 9, 171-178. https://doi.org/10.4236/ojem.2021.94017

Received: September 27, 2021

Accepted: November 2, 2021

Published: November 5, 2021

Copyright $\odot 2021$ by author(s) and Scientific Research Publishing Inc. This work is licensed under the Creative Commons Attribution International License (CC BY 4.0).

http://creativecommons.org/licenses/by/4.0/

\begin{abstract}
Cerebral Venous Sinus Thrombosis (CVST) is blood clot in draining veins and venous sinuses of brain, causing hindrance in the blood drainage system in brain, disturbing the internal homeostasis of brain, resulting in local oedema, ischemia, venous haemorrhage, damage to brain parenchyma and blood brain barrier. In our case report, we discussed a rare presentation of CVST, a 16-year-old young boy who presented in emergency with history recurrent fall, weakness, tingling numbness. What makes it challenging to diagnose in his unusual presentation without common symptoms and on examination no positive neurological finding. This case brings focus on the importance of knowledge about CVST among emergency physicians. CSVT is considered more commonly as a differential diagnosis of stroke in young age group owning to genetic predisposition, hot humid climate of the state leading to severe dehydration, dietary factors leading to vitamin B12 deficiency \& hyper-homocysteinemia etc. Moreover, present COVID-19, inducing a hypercoagulable state among affected individuals gave CVST a new momentous among emergency physicians. An early diagnosis can be very fruitful as it might prevent long term disability and reduce mortality significantly.
\end{abstract}

\section{Keywords}

Cerebral Venous Sinus Thrombosis, Acute Neurological Emergency, Atypical Presentation

\section{Introduction}

Cerebral Venus Sinus Thrombosis (CVST) is a presence of blood clot in the dur- 
al venous sinuses which drains blood from the brain, the cerebral veins or both. The clot keeps the blood from draining out of the brain and as a result of which pressure builds up inside the blood vessels and this can lead to swelling, bleeding in the brain. Cerebral venous thrombosis is a rare stroke presentation in general population affecting mostly young individuals [1] and often turns out to be fatal if not diagnosed early. CVST most commonly presents with severe headache, approximately in $90 \%$ of cases [2]. The incidence of cerebral venous thrombosis (CVT) is estimated at 0.2 to 0.5 per 100,000 population per year [3]. CVT is much more common in women than in men. Ameri and bousser found a female to male ratio of 1.29:1 [4].

CVT is challenging to diagnose because of it is an uncommon neurological emergency. Moreover, it can mimic other disease conditions. Nonetheless it is associated with significant mortality and morbidity which is preventable by early diagnosis and treatment. Symptoms of CVT are difficult to clinically differentiate from other commonly presented conditions. It requires good knowledge about CVT and its risk factors, high index of suspicion, rapid recognition by emergency and primary care physicians to commence early involvement of Neurologists and initiation of definitive treatment for a better outcome and to prevent life threatening complications in CVT patients.

\section{Case of Presentation}

Sixteen-year-old male presented in Emergency Room (ER) with complaints of right sided lower limb weakness which started on previous day of his presentation in ER. Following the development of it he had a domestic fall. He also has been feeling tingling and numbness in his left lower leg since same duration. $\mathrm{He}$ had multiple episodes of fall for last one week. He denied any addiction of any kind. He did not have any comorbidity, neither was allergic to any food or drug etc. He never had COVID-19 before. His vaccination for COVID was still due.

\subsection{On Presentation}

Blood pressure (BP): 110/70 $\mathrm{mmHg}$, heart rate (HR): 64/min, respiratory rate (RR): 20/min, oxygen saturation $\left(\mathrm{SpO}_{2}\right): 97 \%$ Room Air, Temperature: $98.2 \mathrm{~F}$, capillary blood glucose (GRBS): $90 \mathrm{mg} / \mathrm{dl}$.

\subsection{On Neurological Examination}

He was conscious alert oriented. On GlasgowComa Scale his score was 15/15. Power of all four limbs: 5/5. Pupils: Bilaterally (B/L) equal reactive to light. Planters were B/L flexor. No focal neurodeficit was found. Full cranial nerves examination, sensation, coordination, gait was within normal limits.

\subsection{Other Findings}

The cardiopulmonary examination was unremarkable, and he had normal distal pulses in all extremities. His blood reports revealed: elevated levels of serum 
homocysteine $18.14 \mathrm{micromol} / \mathrm{L}$, hemoglobin $17.4 \mathrm{gm} / \mathrm{dl}$, total leucocytes count $9690 /$ cumm, platelet count 224,000/cumm. His 12 leads electrocardiogram was within normal limits.

For further evaluation of his symptoms an MRI brain with venography was diagnosed which revealed CVST involving superior sagittal sinus, straight sinus, left transfers and sigmoid sinuses (Figure 1).

Patient was managed with low molecular weight heparin, oral anti-coagulant (warfarin), anti-epileptic drugs, Tab Homochek containing multivitamins \& minerals, intravenous fluids. Hematologist opinion was taken for? Polycythemiavera (jak2 gene expert was advised).

\section{Literature Review}

Cerebral Venus sinus thrombosis (CVST) is a presence of blood clot leading to complete or partial occlusion in the cerebral venous sinuses (cerebral venous sinus thrombosis) or on smaller cortical veins (cortical vein thrombosis) which drain blood from the brain, the cerebral veins or both. CVST is slightly more common in women, particularly in the age group of 20 to 35, due to pregnancy,

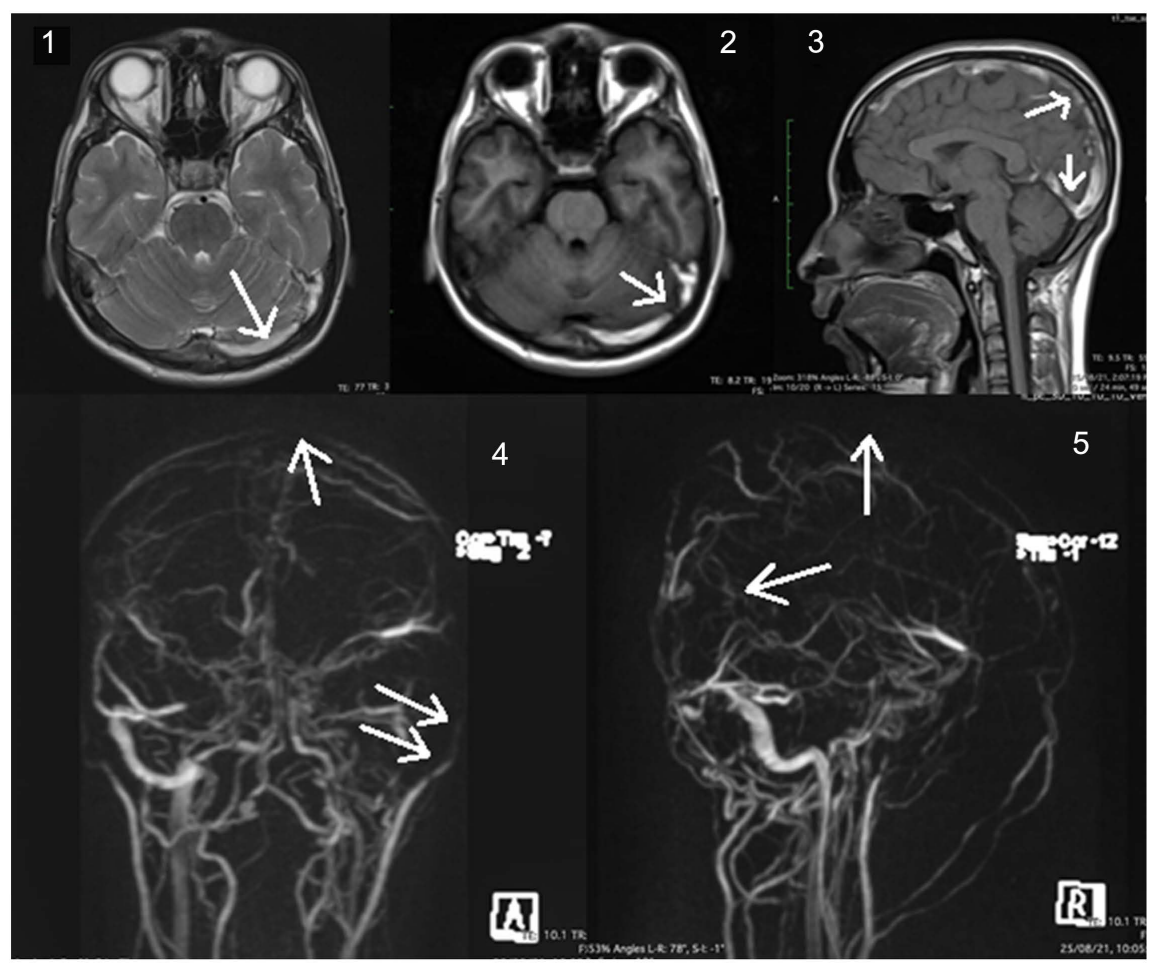

Figure 1. 1: T2 axial image showing loss of flow void in left transverse sinus and sigmoid sinus suggestive of thrombosis. 2: T1 axial section at the level of posterior fossa showing loss of flow void in left transverse sinus and sigmoid sinus appearing hyper intense suggestive of thrombosis. 3: MR venogram sagittal image showing thrombosis of superior sagittal sinus (SSS) and straight sinus as absence of signal in the above mentioned regions. 4: MR venogram coronal section showing non opacification of superior sagittal sinus, straight sinus, left transverse sinus and adjacent left internal jugular vein. 5: T1 segment se image showing SSS and straight sinus thrombosis as hyper intense signals. 
puerperium, and oral contraceptive pills (OCP) use. Mean age in most large studies was between 37 and 38 years though all ages can be affected [5].

\subsection{Risk Factors}

International Study on Cerebral Vein and Dural Sinus Thrombosis found that up to $85 \%$ of adult patients have at least one risk factor; the most common was use of oral contraceptives, followed by a prothrombotic condition (more often genetic than acquired). Ferro et al. [6] mentioned the risk factors of CVST as following:

- Gender/women specific risk factors: use of OCP, pregnancy and puerperium, hormone replacement therapy.

- Systemic conditions: dehydration, obesity malignancy, hyperhomocysteinemia, low vitamin B12 \& folic acid [7] [8] [9], myeloproliferative disorders, inflammatory bowel disease, systemic lupus erythematosus, thyroid disease etc.

- Genetic/acquired thrombotic conditions (thrombophilia): antiphospholipid antibody syndrome, factor V Leiden mutation, antithrombin and protein C \& $S$ deficiency, polycythemia and thrombocythemia.

- Infections: otitis, mastoiditis, sinusitis, meningitis, systemic infection disorders etc.

- Mechanical factors: head injury resulting in direct trauma to the sinuses, lumber puncture and neurosurgical procedures, jugular venous cannulation etc.

- Drugs: ecstasy, lithium, cytotoxic drugs etc.

- Vascular abnormalities: arteriovenous (AV) malformations, Dural AV fistula etc.

CVT is rare but not so rare in the state of Chhattisgarh, India, owning to probable genetic predisposition, hot weather leading to dehydration, unhealthy and stressful lifestyle, addiction in young adults, frequently found hyperhomocysteinemia. Moreover, since the outbreak of novel coronavirus, CSVT has gained particular importance to Emergency Physicians. It has increasingly been reported in association with coronavirus infectious disease of 2019 (COVID-19) owing to its association with hypercoagulability [10].

\subsection{Anatomy}

The dual venous sinuses are veins located between the two layers of dura matter, i.e. endosteal and meningeal layer. They are valveless allowing bidirectional blood flow from and into intracranial veins and run alone and not along the arteries. They can be grossly classified as:

Middle unpaired sinuses: superior sagittal sinus, inferior sagittal sinus, straight sinus, occipital sinus, intercave nous sinus.

Paired sinuses (drains into the middle vessels): transverse sinus, sigmoid sinus, superior \& inferior petrosal sinus, cavernous sinus, sapheno-parietal sinus, basilar venous plexus. 
Together with the Dural venous sinuses form the major drainage system from brain towards the internal jugular veins via sigmoid sinuses [11] (Figure 2).

\subsection{Pathophysiology}

1) Thrombosis of cerebral vein: leading to increased venular \& capillary pressure.

2) Thrombosis of major cerebral venous sinus: decreased CSF absorption.

There after either of them eventuates in raised intracranial pressure, cytotoxic $\&$ vasogenic edema, ultimately disruption of blood brain barrier leads to parenchymal tissue damage. A continuous rise in pressure results in capillary hypertension, cerebral edema, and venous hemorrhagehe [12].

\subsection{Clinical Presentation}

CVST can manifest with a wide range of symptoms and sometimes its presentation depends on the site and extent of thrombosis. Headache being the commonest clinical symptom described as throbbing (9\%), band like (20\%), thunderclap (5\%) masquerading subarachnoid hemorrhage, and other (pounding, exploding, stabbing, etc.) (20\%) by Wasay et al. in their study. Farb et al. found that CVST may also present pseudo tumorcerebri-like syndrome. Other symptoms \& signs may include altered sensorium, hemiparesis or limb weakness, aphasia, ataxia, dizziness, chorea, and cranial nerve syndromes (vestibular neuropathy, pulsatile tinnitus, unilateral deafness, double vision, facial weakness, and obscuration of vision), seizure etc. It is worth mentioning that though CVST is rare but not so rare in the state of Chhattisgarh, India, owing to genetic predisposition, it's hot dry climate leading to dehydration, addiction in young adults, strict vegetarian or unbalanced dietary habit leading to nutritional deficiencies.

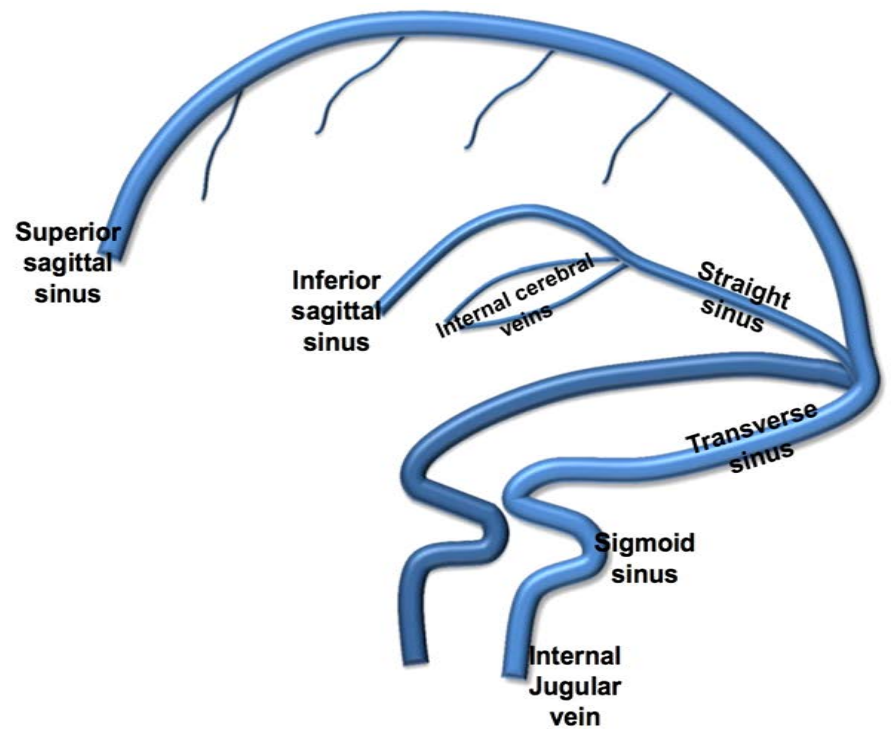

Figure 2. Cerebral venous system components [14]. 


\subsection{Diagnosis \& Management}

Identifying the potentiality of CSVT is the utmost important step in diagnosis which requires high index of suspicion by emergency physicians and other clinicians, and that only comes with good knowledge regarding the diverse presentation of CSVT. Non contrast enhanced CT scan of brain (cord sign), contrast enhanced CT brain (empty delta sign), CT/MR venography and lastly CT/MR brain angiography (corscrew appearance of veins) are mainstay diagnostics. Once CSVT diagnosis is established, or even in cases of high suspicions, neurologist or primary care physician needs to investigate for underlying risk factors.

\subsection{Treatment}

Focuses on primary stabilization of the patient followed by initiation of anti-thrombotic treatment with heparin or low molecular weight heparin at earliest which then to be converted to oral anti-coagulant (warfarin or direct factor Xa inhibitors). Even if there is presence of hemorrhagic infarct most clinicians start heparin straightaway after the diagnosis of CVST is made, as clinical trials show no increased or new cerebral hemorrhages developing after treatment with heparin [13]. Other treatment modalities include thrombolysis/direct thrombectomy, management of sequel (seizures, raised intracranial pressure and venous infection), prevention of cerebral herniation, and prevention of relapse.

\section{Discussion}

In our case, a young male patient presented to ER without any common presentation of CVST commonest being headache. Rather this boy presented in ER with a history of recurrent fall, weakness, tingling and numbness without any positive neurological finding during examination making it extremely challenging to consider CVST as a differential diagnosis in the first place and difficult to discern from other more common neurological conditions. In our present case scenario the detailed history about recurrent fall and unilateral non traumatic lower limb weakness raised a suspicion about CVST. Hence MRI brain with venography was done which revealed CVST involving superior sagittal sinus, straight sinus, left transfers sinus and sigmoid sinuses.

CVST is a rare neurological emergency globally. Though in the state of Chhattisgarh CVST not so uncommon owning to its hot and humid climate leads to severe dehydration, extensive use of OCPs, genetic predisposition, hypercoagulable state, dietary habit leads to deficiency of vitamin B12 and hyperhomocysteinemia etc. Moreover in present COVID era, COVID-19 and post COVID state both being hypercoagulable states, CVST gained a whole new importance as an Emergency Room differential diagnosis making us, the emergency physicians more often considering its possibility in their patients than ever before.

Although the diagnosis was made but the underlying etiology was yet to be found and in the search of the underlying cause that leads to CVST in such a 
young adult we did thorough investigation for the causative factors that could have possibly been related to. Our patient's blood reports revealed: elevated levels of serum homocysteine $18.14 \mathrm{micromol} / \mathrm{L}$, hemoglobin $17.4 \mathrm{gm} / \mathrm{dl}$ more than normal level, total leucocytes count $9690 /$ cumm and platelet count $224,000 /$ cumm were within normal limits. In his case, Hyperhomocystenemia being a surrogate marker of vitamin B12 deficiency which is a risk factor CVST could be the leading cause of CVST in such a young individual. Therefore we corrected vitamin B12 deficiency \& hyperhomocystemia in our patient with oral supplements along with anticoagulants. It is worth to mention that abnormally raised hemoglobin level alarmed us as CVST has a wide range of etiology and polycythemia is one of them. Hence, we also investigated our patient for polycythemia vera by JAK2 gene mutation study which found to be negative in his case. Though it could not totally rule out the possibility of polycythemia vera as cases of recurrent CVST have been reported in young male with JAK2 negative polycythemia vera15 in previous literatures that could have been a possibility in our patient as well.

Fortunately our patient was diagnosed early as we the emergency physicians in the state of Chhattisgarh always keep a high threshold for CVST in all neurological emergencies. Early initiation of treatment resulted in rapid recovery and he was discharged in a stable condition thereafter. During follow up visits he reported no recurrence of his symptoms.

\section{Conclusion}

Almost $80 \%$ of patient with CVT recovers without functional disability if diagnosed \& treated early. However, it requires high index of suspicious and good clinical knowledge to diagnose it in the first place as the range of clinical signs and symptoms vary, may evolve suddenly or over the weeks, mimicking many other neurological conditions. So, emergency \& primary care physicians must keep CVST as a differential diagnosis in cases with acute neurological presentations in emergency mostly in young individuals. CVST gained special importance after the introduction of COVID-19 pandemic worldwide. Creating awareness about CVST among emergency physicians will lead to early diagnosis and prompt treatment. Appropriate therapy will reverse the disease progression and significantly reduce the risk of acute complications.

\section{Declaration}

Signed informed consent obtained from the patient and guardians with no intentions harm to human subjects and animal. This case report is purely for learning purpose.

\section{Conflicts of Interest}

The authors declare no conflicts of interest regarding the publication of this paper. 


\section{References}

[1] Ruiz-Sandoval, J.L., Chiquete, E., Bañuelos-Becerra, L.J., Torres-Anguiano, C., González-Padilla, C., Arauz, A., et al. (2012) Cerebral Venous Thrombosis in a Mexican Multicenter Registry of Acute Cerebrovascular Disease: The RENAMEVASC Study. Journal of Stroke and Cerebrovascular Diseases, 21, 395-400. https://doi.org/10.1016/j.jstrokecerebrovasdis.2011.01.001

[2] Bousser, M.G. and Ferro, J.M. (2007) Cerebral Venous Thrombosis: An Update. Lancet Neurology, 6, 162-170. https://doi.org/10.1016/S1474-4422(07)70029-7

[3] Stam, J. (2005) Thrombosis of the Cerebral Veins and Sinuses. New England Journal of Medicine, 352, 1791-1798. https://doi.org/10.1056/NEJMra042354

[4] Ameri, A. and Bousser, M.G. (1992) Cerebral Venous Thrombosis. Neurologic Clinics, 10, 87-111. https://doi.org/10.1016/S0733-8619(18)30235-4

[5] Allroggen, H. and Abbott, R.J. (2000) Cerebral Venous Sinus Thrombosis. Postgraduate Medical Journal, 76, 12-15. https://doi.org/10.1136/pmj.76.891.12

[6] Ferro, J.M., Canhao, P., Stam, J., Bousser, M.-G. and Barinagarrementeria, F. (2004) Prognosis of Cerebral Vein and Dural Sinus Thrombosis: Results of the International Study on Cerebral Vein and Dural Sinus Thrombosis (ISCVT). Stroke, 35, 664-670. https://doi.org/10.1161/01.STR.0000117571.76197.26

[7] Yoo, J.H., Chung, C.S. and Kang, S.S. (1998) Relation of Plasma Homocyst(e)ine to Cerebral Infarction and Cerebral Atherosclerosis. Stroke, 29, 2478-2483. https://doi.org/10.1161/01.STR.29.12.2478

[8] Toole, J.F., Malinow, M.R., Chambless, L.E., Spence, J.D., Pettigrew, L.C., Howard V.J., et al. (2004) Lowering Homocysteine in Patients with Ischemic Stroke to Prevent Recurrent Stroke, Myocardial Infarction, and Death: The Vitamin Intervention for Stroke Prevention (VISP) Randomized Controlled Trial. JAMA, 291, 565-575. https://doi.org/10.1001/jama.291.5.565

[9] Saposnik, G., Ray, J.G., Sheridan, P., McQueen, M. and Lonn, E. (2009) Homocysteine-Lowering Therapy and Stroke Risk, Severity, and Disability: Additional Findings from the HOPE 2 Trial. Stroke, 40, 1365-1372.

https://doi.org/10.1161/STROKEAHA.108.529503

[10] Ghosh, R., Roy, D., Mandal, A., Pal, S.K., Chandra Swaika, B., Naga, D., Pandit, A., Ray, B.K. and Benito-León, J. (2021) Cerebral Venous Thrombosis in COVID-19. Diabetes \& Metabolic Syndrome: Clinical Research \& Reviews, 15, 1039-1045. https://doi.org/10.1016/j.dsx.2021.04.026

[11] Gaillard, F. and Hacking, C. (2021) Dural Venous Sinuses. https://doi.org/10.53347/rID-5052

[12] Ungersböck, K., Heimann, A. and Kempski, O. (1993) Cerebral Blood Flow Alterations in a Rat Model of Cerebral Sinus Thrombosis. Stroke, 24, 563-569. https://doi.org/10.1161/01.STR.24.4.563

[13] Celorrio, C.S.Y., Palma, B.L. and Rodríguez, P.L.R. (2018) Cerebral Venous Thrombosis. Revista Cubana de Neurología y Neurocirugía, 8, 1-23.

[14] Thorell, S.E., Parry-Jones, A.R., Punter, M., Hurford, R. and Thachil, J. (2015) Cerebral Venous Thrombosis-A Primer for the Haematologist. Blood Reviews, 29, 45-50. https://doi.org/10.1016/j.blre.2014.09.006 